\title{
OTIMIZAÇÃO DO USO DOS ENXERTOS PANCREÁTICOS NO BRASIL
}

\author{
Optimization the use of pancreatic grafts in Brazil
}

Paolo Rogério de Oliveira Salvalaggio', Marcelo Perosa² e José Maria Figueiró ${ }^{3}$

\begin{abstract}
RESUMO
No B rasil e no mundo usa-se de forma mais adequada o número de doadores disponíveis para receptores de rim e de fígado do que para receptores de pâncreas ou ilhotas de L angerhans. Esta revisão tem como objetivo demonstrar resultados atuais com o uso de doadores não ideais de pâncreas, para que se possa aumentar o aproveitamento deste órgão para transplante no Brasil. Com uso de critério mais abrangente para aceitação de doadores, podem-se incluir para doação do pâncreas: doadores com exames laboratoriais alterados, doadores com anormalidades vasculares, doadores pediátricos, doadores "idosos", doadores obesos, doadores com coração parado, doadores que recebem drogas vasoativas e doadores com malformação pancreático-biliar congênita. Não se devem descartar potenciais doadores de pâncreas baseando-se somente na idade, peso ou com base em exames laboratoriais e uso de drogas vasoativas no doador. M ais importante, a inspeção e pal pação do órgão por um cirurgião experiente será o fator determinante final do possível uso do pâncreas para transplante de pâncreas ou de il hotas de $L$ angerhans. A normal idades vasculares, assi $m$ como a doação do fígado e do intestino delgado não devem contraindicar a doação do pâncreas. Presença de gordura, fibrose e aterosclerose são as características que devem ser levadas em consideração quando do uso de doadores não ideais para transplante de pâncreas. Ó rgãos que não possam ser utilizados para transplante de pâncreas devem ser considerados para isolamento de il hotas de Langerhans.
\end{abstract}

Descritores: doadores, transplante de pâncreas, il hotas de L angerhans, enxerto pancreático, B rasil

Grau acadêmico:

1. Professor de Cirurgia, Divisão de Transplantes de Múltiplos Órgãos, Saint Louis University, St. Louis, MO, USA.

2. Cirurgião do Departamento de Cirurgia do Hospital da Beneficiência Portuguesa, São Paulo, SP.

3. Cirurgião do Departamento de Cirurgia do Hospital Felicio Rocho, Belo Horizonte, MG.

Instituição:

Department of Surgery - Division of Abdominal Organ Transplantation

Saint Louis University

Correspondência:

Dr. Paolo Rogério de Oliveira Salvalaggio

3635 Vista Avenue, St Louis, M0, 63110

Fone : 0013142685280 Fax: 0013145778714

E-mail: psalvala@slu.edu

Recebido em: 18/10/2004

Aceito em: 30/09/2005

\section{INTRODUÇÃO}

Diabetes melittus (DM) afeta $6 \%$ da população mundial, gerando complicações vasculares que a caracterizam como a oitava causa de morte nos Estados U nidos e a maior causa de insuficiência renal crônica e cegueira em adultos, bem como a maior causa deamputações de membros inferiores e impotência sexual no mundo. ${ }^{1}$

Q uase um século após sua descoberta, a terapia com insul ina exógena permite controle das complicações metabólicas agudas do DM . No entanto, as complicações crônicas da doença ai nda ocorrem em um número significativo de pacientes. Em 1993, foram examinados os efeitos do controle intensivo da glicemia, relacionando-o com a gravidade e freqüência das complicações do D M. Concluiu-se que a terapia intensiva de manutenção de normoglicemia em pacientes diabéticos do tipo I retarda 0 aparecimento e 0 desenvolvimento das complicações da doença, ${ }^{1}$ apesar de aumentar em duas a três vezes o número de episódios graves de hipoglicemia e ter baixo índice de adesão dos pacientes.

0 primeiro transplante pancreático foi realizado pelo grupo da Universidade de M innesota, em 1966. ${ }^{2}$ Em 2000, o grupo da Universidade de Edmonton foi o primeiro a descrever resultados satisfatórios em longo prazo com o uso de transplantes de ilhotas de $L$ angerhans em humanos. ${ }^{3}$

A tual mente, o transplante de pâncreas ou de ilhotas pancreáticas são as únicas modalidades terapêuticas capazes de controlar fisiologicamente a glicemia e a insulinemia através da reposição do tecido pancreático endócrino. A tual mente, segundo dados do Registro Internacional de Transplante de Pâncreas e I lhotas, cerca 
de 20.000 transplantes de pâncreas (mais de 1.800/ano entre 20012003) e mais de 700 transplantes de il hotas de L angerhans jáforam realizados no mundo. ${ }^{4,5}$

0 transplante de pâncreas vem apresentando resultados excelentes em centros de excelência, com baixa mortalidade ci rúrgica e progressiva melhora da sobrevida do enxerto, proporcionando insulinoindependência e melhora na qualidade de vida aos pacientes. ${ }^{6-9}$

A lém de bons resultados, uma maior atenção dos profissionais de saúde e conhecimento público a respeito deste tratamento vem trazendo um maior número de pacientes a buscar o transplante como tratamento definitivo do DM no Brasil e no mundo. Conseqüentemente, está ocorrendo um aumento no número de diabéticos em lista de espera e o tempo em lista vem aumentando a cada ano devido à escassez de órgãos disponíveis para transplante..10,11

Os diabéticos enfrentam um problema maior na lista de espera. A pesar de uma mortalidade maior, existe um menor número de doadores que estejam dentre os critérios usuais para doação de pâncreas ou de ilhotas de Langerhans. A Igumas publicações recentes chamaram a atenção para 0 sub-uso de doadores de pâncreas nos Estados Unidos: apenas 22\% do total de doadores cederam o pâncreas, $40 \%$ foram doadores de coração, enquanto 84\% destes foram doadores de fígado (índice de um pâncreas: 3.26 fígados). ${ }^{10-12} \mathrm{Na}$ Europa, um estudo recente demonstrou que apenas $10-28 \%$ do total de doadores podem ser utilizados para transplante de pâncreas, com um adicional de 31-38\% para transplante de ilhotas de $L$ angerhans. De maneira interessante, receptores de transplante de pâncreas ou de il hotas de $L$ angerhans não necessitam competir pelos mesmos doadores se critérios claros forem seguidos. ${ }^{13} 0$ índice de doadores de pâncreas/fígado no mundo é ainda pior 1:7.2. ${ }^{11}$

No Brasil, a situação é semel hante. Embora não existam dados oficiais disponíveis do número de doadores falecidos, sabe-se que foram realizados 1504 transplantes de rins e 615 transplantes de fígado no ano de 2003. Neste mesmo período, somente 212 transplantes de pâncreas foram real izados (índice de um pâncreas: 2.9 fígados). ${ }^{14}$ Certamente, um mel hor sistema de captação, alocação e distribuição de órgãos, bem como educação da população e da comunidade médica, podem contribuir para um aumento substancial do número de doadores e, conseqüentemente, de transplante de pâncreas no nosso país.

No entanto, parece que usamos de forma mais adequada o número de doadores disponíveis para receptores de rins efígados, existindo desta maneira um sub-uso de doadores de pâncreas no B rasil. Esta revisão tem como objetivo demonstrar resultados atuais com o uso de doadores não ideais de pâncreas, para que se possa incrementar o número de doadores de pâncreas no Brasil.

\section{EXPANSÃO DO CRITÉRIO DE DOADORES PARA TRANSPLANTE DE PÂNCREAS E ILHOTAS DE LANGERHANS: USO DE DOADORES NÃO IDEAIS}

0 uso de doadores não ideais vem sendo realizado há alguns anos para transplante renal e mais recentemente hepático, com dados escassos em transplante pancreático. ${ }^{10,11,13,15-17}$

E $m$ transplante de pâncreas, um critério mais abrangente para aceitação de doadores incluiria o uso de: doadores com exames laboratoriais alterados, doadores com anormalidades vasculares hepato-pancreáticas, doadores pediátricos, doadores "idosos", doadores obesos, doadores com coração parado ("non-heart beating donors"), doadores que recebem drogas vasoativas e doadores com malformação pancreático-biliar congênita. M ais importante, o critério de aceitação não se restringe somente a valores laboratoriais, idade, peso e condição clínica do doador, mas principalmente a uma avaliação macroscópica meticulosa (inspeção e palpação) durante a retirada do pâncreas do doador falecido, por um cirurgião transplantador experiente. A inda assim, história clínica de DM ou intolerância à glicose, trauma grave ao pâncreas ou duodeno, evidência macroscópica de pancreatite aguda grave com saponificação, hemorragia e/ou necrose persistem como contra-indicações absolutas à doação de pâncreas.

Com o uso de um critério mais abrangente, o grupo de W isconsin conseguiu ampliar a captação de doadores pancreáticos com um índice de um pâncreas para cada 1.25 fígados. ${ }^{11}$ Conseguiu-se dobrar o número de doadores de pâncreas com a utilização de doadores não ideais. ${ }^{18} 0$ aumento no número de doadores também já foi relatado pelo grupo de Pittsburgh e de L eipzig (A lemanha). ${ }^{16,19,20}$ No B rasil, já existe relato também de resultados semel hantes. ${ }^{21}$

\section{USO DE DOADORES COM EXAMES LABORATORIAIS ALTERADOS}

Estudos do final da década de 80 comprovaram que pacientes com traumatismo craniano apresentam hiperamilasemia. $22,23,24$ Isto não se correlaciona com a função endócrina do pâncreas e não altera os resultados do transplante. Da mesma maneira, não existem estudos que utilizem lipase como fator determinante de função endócrina do pâncreas ${ }^{22-24}$. Caso ainda haja suspeita de pancreatite aguda ou trauma pancreático, uma tomografia computadorizada pode ajudar a avaliar possível lesão no pâncreas. Pesquisas antigas revel aram uma relação entre hipergl icemia do doador (sem história clínica de DM ou intolerância à glicose) e sobrevida do enxerto, o que na época, reduzia a quantidade de doadores de pâncreas em até $50 \% .{ }^{22-24} \mathrm{Com}$ a evolução das técnicas cirúrgicas e o manejo da imunossupressão na década de 90 , conseguiu-se demonstrar que a hiperglicemia no doador não aumenta os riscos de rejeição no receptor e não representa uma redução funcional na massa de células $\beta$ no doador. Comprovouse que a hiperglicemia do doador não se relaciona diretamente com dano à função pancreática endócrina. A ssi m sendo, doadores com hipergl icemia não apresentam nenhuma alteração histológica no pâncreas e freqüentemente recebem corticóides e/ou solução glicosada. ${ }^{25}$ A ssim, a hiperglicemia nada mais representa que uma resposta fisiológica do organismo ao trauma, resultante da ação de hormônios contra-reguladores (glucagon, cortisol e catecolaminas) para a esti mulação da gluconeogênese hepática. ${ }^{25}$ Portanto, não se devem descartar potenciais doadores de pâncreas baseando-se somente em exames laboratoriais. A inspeção e a palpação do órgão por um cirurgião experiente será o fator determinante final do possível uso do pâncreas de um doador. A inda parece prudente a utilização de insulina no manejo do potencial doador para que o mesmo seja mantido em um estado euglicêmico e, desta forma, se evite um maior "stress" sobre as il hotas de L angerhans. ${ }^{22-24}$

\section{USO DE DOADORES COM ANORMALIDADES VASCULARES HEPATO-PANCREÁTICAS}

Há mais de uma década foi demonstrado que o mesmo doador pode ceder o fígado e 0 pâncreas para diferentes receptores. ${ }^{26,27}$ Posteriormente, estudos comprovaram que anomalias vasculares 
não alteram a sobrevida do enxerto pancreático ou hepático. $28-30$ A lgumas anormalidades vasculares de fato podem, inclusive, facilitar a remoção do pâncreas do doador ou a reconstrução ex vivo. ${ }^{31-33}$ Parece ainda que a remoção em bloco do pâncreas e do fígado pode facilitar a cirurgia do doador ou encurtar a duração da mesma. ${ }^{34-38} \mathrm{M}$ ais recentemente, demonstrou-se que o mesmo doador pode ainda ceder o intestino delgado para transplante em um diferente receptor, desde que haja uma coordenação apropriada entre as diferentes equipes envolvidas na cirurgia do doador. ${ }^{34-38}$ A ssim, as anormalidades vasculares não devem contra-indicar de maneira nenhuma a doação do pâncreas, mas, ao mesmo tempo, desafiam os cirurgiões para que reconstruam o suprimento arterial do pâncreas de maneira adequada, usando criatividade e princípios básicos de cirurgia vascular, mantendo sempre o órgão imerso em solução de preservação a baixa temperatura e fazendo uso de "Iupas" cirúrgicas de aumento, iluminação e instrumental cirúrgico adequado.

\section{USO DE DOADORES PEDIÁTRICOS}

D oadores pediátricos são usados raramente para transplante de pâncreas e também já foram descritos para uso em transplante de ilhotas de L angerhans..$^{34-38} 0$ limite inferior de idade considerado não ideal ainda permanece indefinido, variando de sete a 15 anos. ${ }^{20,34-39} \mathrm{~A}$ I guns fatores técnicos podem contribuir para a obtenção de um bom resultado: a) se possível, o tronco celíaco e a artéria mesentérica superior devem ser retirados em bloco com um "patch" da aorta abdominal, b) um receptor de superfície corporal pequena deve ser selecionado, principal mente para que seja maximizada a massa glomerular em casos de transplante duplo de rim e pâncreas e c) mais importante do que o tamanho do pâncreas e limites de peso ou idade do doador será o diâmetro dos vasos que suprirão sua vascularização arterial. ${ }^{20} \mathrm{~A}$ ssim, a criatividade, a experiência e a disponibilidade de cirurgião treinado em reconstrucões vasculares e em transplante pancreático é de importância suprema no uso destes doadores. Vale ainda ressaltar que, com vasos de diâmetro reduzido e um órgão com alta resistência vascular, o risco de trombose vascular será certamente aumentado.

\section{USO DE DOADORES “IDOSOS"}

Da mesma forma que para doadores pediátricos, o limite de idade do qual se considera que um doador não seria ideal ai nda permanece indefinido, variando de 40-55 anos. ${ }^{20,} 39$ De maneira paradoxal, existe, ao mesmo tempo, um aumento do número de doadores com o diagnóstico de acidente vascular cerebral e uma diminuição do número de doadores com trauma encefálico. D esta forma, há uma tendência de aumento da idade média dos doadores. Com o uso de um critério conservador para utilização do doador de pâncreas, deve ocorrer uma perda significativa de órgãos para transplante, situação esta que pode se tornar mais crítica com o passar dos anos.

Infelizmente, os resultados de transplante pancreático com a utilização de doadores idosos não são tão satisfatórios. 0 doador de pâncreas, com idade acima de 45 anos, determina aumento consistente no número de complicações técnicas pós-transplante, tais como trombose vascular, fístulas e sangramento pós-operatório. Perda precoce e/ou disfunção inicial do enxerto pancreático, utilização prolongada de insulina, aumento no risco de re-operações, de infecções de ferida intra-abdominal, nos custos do transplante de pâncreas e na sobrevida em longo prazo do enxerto também já foram descritos. ${ }^{15} \mathrm{Um}$ estudo do grupo de M inneapolis revelou potencial aumento da mortalidade perioperatória com a utilização de doadores idosos. ${ }^{15}$ Já foi descrito o uso de doadores idosos com resultados similares aos de doadores ideais em termos de função do al oenxerto e independência de insulina, índice de complicações, bem como sobrevida média dos pacientes pós-transplante. ${ }^{15} 0$ grupo de Pittsburgh também relatou resultados similares (sobrevida do enxerto e do paciente e índice de complicações) aos de doadores ideais, embora com um maior índice de disfunção do enxerto.

A ssim, parece que, numa era de carência de órgãos para transplante e aumento do número de pacientes e do tempo em lista de espera, estabelecer idade absoluta máxima como critério de exclusão de doador de pâncreas não parece prudente. M ais importante, a inspeção e palpação do órgão e dos vasos por um cirurgião experiente será o fator determinante final do possível uso do pâncreas de um doador. Presença de gordura subcapsular, fibrose, aterosclerose arterial, função renal (em caso de transplante duplo de pâncreas e rim) e de outros dados clínicos relevantes no doador, bem como uma seleção apropriada de um receptor com menor número de fatores de risco de complicações são as características que devem ser levadas em consi deração quando do uso de doadores idosos para transplante de pâncreas.

\section{USO DE DOADORES OBESOS}

Também não existe consenso do limite máximo de peso de um doador de pâncreas. N úmeros variam entre 100 quilos, ou índice de massa corporal entre 27-35. Sabe-se que, com o aumento do índice de massa corporal, há um aumento também progressivo na não utilização de doadores para transplante de pâncreas. ${ }^{10,19,40}$ Por outro lado, há um aumento da prevalência de sobrepeso ou obesidade na população mundial. A ssim como no uso de doadores idosos, o uso de critério conservador para utilização do doador de pâncreas pode gerar perda significativa de órgãos para transplante.

O s resul tados de transplante pancreático com a utilização de doadores obesos também não são tão satisfatórios. Há aumento no número de complicações técnicas pós-transplante, principalmente relacionadas a infecções intra-abdominais. ${ }^{11}{ }^{41} \mathrm{~N}$ o entanto, não existem estudos recentes relacionando o uso de pâncreas com infiltração gordurosa e pior resultado com transplante de pâncreas.

Estudo da Universidade de Pittsburgh descreveu características histológicas de pâncreas com infiltração gordurosa obtidas de doadores que não foram utilizados para transplante. Os autores descrevem uma opção interessante de realizar lipectomia da glândula, desde que não haja trauma ao parênquima e ao sistema ductal pancreático. ${ }^{19}$ O utra opção que está atraindo atenção da comunidade transplantadora é a utilização de pâncreas com infiltração gordurosa para transplante de ilhotas de Langerhans. Parece que, nestas condições, a gordura pode facilitar a digestão da glândula no processo de purificação de ilhotas de Langerhans, ao mesmo tempo em que parece conter maior número de ilhotas de Langerhans.

\section{USO DE DOADORES COM CORAÇÃO PARADO ("NON- HEART BEATING DONORS", "DONATION AFTER CARDIAC DEATH")}

O utra opção para o aumento do número de doadores para transplante é a utilização de doadores com coração parado ("non-heart beating donors, donation after cardiac death") . A legisl ação brasileira ainda 
não se posicionou claramente quanto à utilização destes pacientes como doadores de órgãos. Cabe à comunidade transplantadora lutar para que mais esta opção seja oferecida a milhares de brasileiros em fila de espera.

R elatos de transplante pancreático com a utilização destes doadores ainda são escassos na literatura. No entanto, parece que a utilização destes doadores pode gerar resultado funcional comparável a doadores ideais de pâncreas. ${ }^{42-44}$ Estes doadores também podem ser utilizados para transplante de ilhotas de Langerhans..$^{45}$ Deve-se evitar o uso de doadores com tempo de isquemia quente maior que 30 minutos e que tenham outros fatores de risco para disfunção do enxerto.

\section{USO DE DOADORES QUE RECEBEM DROGAS VASOATIVAS}

O s mesmos estudos i niciais que associaram hiperglicemia à al teração na função do enxerto pancreático não demonstraram correlação entre uso de drogas vasoativas (dopamina) e funcionamento do enxerto pancreático. ${ }^{21,46-48}$ A ssim, o uso de drogas vasoativas não deve contraindicar o uso do pâncreas do doador para transplante. Cuidado adicional deve ser tomado em pacientes com instabilidade hemodi nâmica e uso de múltiplas e/ou altas doses de drogas vasoativas. Novamente, a inspeção e palpação do órgão por um cirurgião experiente será o fator determinante final do possível uso do pâncreas de um doador. Em determinadas situações, a dissecção in situ do doador deve ser minimizada para que a solução de preservação possa ser utilizada rapidamente e se evite isquemia ao enxerto. Importante também é considerar a futura função do enxerto renal em casos de transplante duplos de rim e pâncreas. 0 rim parece ser mais suscetível ao uso de drogas vasoativas no doador e, certamente, existe um maior risco de disfunção do enxerto com necessidade do uso de diálise prolongada no período pós-operatório. D esta forma, deve-se sempre informar aos receptores sobre estes riscos e aval iar o risco e o benefício da utilização de doadores não ideais nestas situações.

\section{USO DE DOADORES COM MÁ FORMAÇÃO PANCREÁTICO-BILIAR CONGÊNITA}

Existem dois relatos na literatura do uso com sucesso de pâncreas anular, sem obstrução duodenal, para transplante. ${ }^{49,50}$ Embora isto não seja uma situação corriqueira, parece adequado que se individualize caso a caso e não se exclua o potencial doador somente por apresentar uma anormalidade congênita. 0 mesmo parece válido para potenciais receptores de transplante duplo, onde 0 doador apresenta rim em ferradura sem história prévia de complicações urológicas ${ }^{51}$. Desta maneira, julgamos que se possa maximizar o potencial número de doadores e fazer com que cada doador extra possa beneficiar múltiplos receptores.

\section{USO DE DOADORES PARA TRANSPLANTE DE ILHOTAS DE LANGERHANS}

É possível que, depois da recente real ização do primeiro transplante de ilhotas de L angerhans no B rasil, o procedimento aumenteem número no nosso meio nos próximos anos. ${ }^{52} \mathrm{~A}$ utilização de pâncreas de doadores não ideais, especial mentedaqueles com infiltração gordurosa podefazer parte de uma estratégia para que todos os órgãos de um potencial doador sejam utilizados para pacientes em lista de espera.

$\mathrm{Na}$ A mérica do Norte, receptores detransplante depâncreas ou deil hotas deL angerhans ainda não competem pel os mesmos órgãos. Os primeiros têm preferência no atual sistema de alocação. Com uma melhora dos resultados do transplante de ilhotas de L angerhans esta prática provavel mente seja revisada. U m estudo de um grupo suiço demonstrou que ambos os procedimentos não são mutuamente exclusivos e não há necessidade de competição pelos mesmos doadores. Pelo contrário, o estabelecimento de critérios claros fez com que houvesse uma maior captação de pâncreas para transplante. ${ }^{13}$

\section{CONCLUSÕES}

H istória clínica de diabetes ou intolerância à glucose, trauma severo ao pâncreas ou duodeno, evidência macroscópica de pancreatite aguda severa com saponificação, hemorragia e/ou necrose permanecem ainda como contra-indicações à doação do pâncreas. Não se devem descartar potenciais doadores de pâncreas baseando-se somente na idade, peso (ou massa corporal) ou com base em exames laboratoriais e uso de drogas vasoativas no doador. M ais importante, a inspeção e pal pação do órgão por um cirurgião experiente será o fator determinantefinal do possível uso do pâncreas para transplante de pâncreas ou de ilhotas de L angerhans. D urante a cirurgia do doador, anormalidades vasculares não devem contra-indicar de manei ra nenhuma a doação do pâncreas, bem como a doação do fígado e do intestino del gado não impossibilitam a doação do pâncreas. Presença de gordura subcapsular, fibrose, aterosclerose arterial, função renal (em caso de transplante duplo de pâncreas e rim) no doador, bem como uma seleção apropriada de um receptor com menor número defatores de risco de complicações são as características que devem ser levadas em consideração quando do uso de doadores não ideais para transplante de pâncreas. Ó rgãos que não possam ser utilizados para transplante de pâncreas devem ser sempre considerados para isolamento de il hotas de $L$ angerhans. E m época de falta de órgãos para transplante e aumento do número de pacientes e tempo em lista de espera, profissionais da saúde envolvidos com transplante devem conhecer riscos e resultados com o uso de doadores não ideais para transplante de pâncreas e de ilhotas de L angerhans. Uma atitude mais agressiva, que leve em consideração os dados publicados na literatura mundial, poderá aumentar consideravel menteo número de doadores e, conseqüentemente, detransplantes de pâncreas e de ilhotas de L angerhans em nosso país.

\section{ABSTRACT}

Liver and kidney grafts have been more optimized than pancreas grafts worldwide. This review aims to show strategies of using nonideal pancreatic donors in order to increase the amount of organs for pancreas transplantation in B razil. Non-ideal donors for pancreas transplantation include those with hyperamylasemia, hyperglycemia, using vasopressors, and the pediatric, old, obese and non-heart-beating donors, as well as donors with vascular abnormality or donors with congenital abnormalities in the graft. Donors should not be excluded based only on their age, weight, blood tests or due to the use of vasoactive drugs. I mportantly, the inspection and palpation of the pancreas by an experienced surgeon will be the determinant factor to the feasibility for the organ donation for islet or pancreas transplantation. During the donor surgery, vascular abnormalities as well as the simultaneous donation of the liver and/or the small bowel should not
} 
preclude the pancreas donation. The presence of fat infiltration, fibrosis, atherosclerosis, and the renal function of the donor, as well as a proper selection of the recipient are the main factors to be used to determine whether marginal donors will be used or not for the pancreas or islet transplantation. Organs that should not be used for pancreas transplantation might be used for islet transplantation. A $n$ aggressive policy can significantly raise the number of pancreas available for transplantation in our country.

Key-words: donors, pancreas transplantation, islets, pancreatic grafts, B razil.

\section{REFERÊNCIA}

1. The effect of intensive treatment of diabetes on the development and progression of long-term complications in insulin-dependent diabetes mellitus. The Diabetes Control and Complications Trial Research Group. N Engl J Med 1993;329(14):977-86.

2. Kelly WD, Lillehei RC, Merkel FK, al. e. Allotransplantation of the pancreas and duodenum along with the kidney in diabetic nephropathy. Surgery 1967;61(6):827-37.

3. Shapiro AM, Lakey JR, Ryan EA, Korbutt GS, Toth E, Warnock GL, et al. Islet transplantation in seven patients with type 1 diabetes mellitus using a glucocorticoidfree immunosuppressive regimen. N Engl J Med 2000;343(4):230-8.

4. Gruessner A, Sutherland DE, Gruessner RW. Report of the International Pancreas Transplant Registry (IPTR). Available at http://www.iptr.umn.edu/; 2004

5. Hering B. Report of the International Islet Transplant Registry (IITR). Available at http://www.med.uni-giessen.de/itr/; 2001.

6. Farney AC, Cho E, Schweitzer EJ, Dunkin B, Philosophe B, Colonna J, et al Simultaneous cadaver pancreas living-donor kidney transplantation: a new approach for the type 1 diabetic uremic patient. Ann Surg 2000;232(5):696-703.

7. Kaufman DB, Leventhal JR, Koffron AJ, Gallon LG, Parker MA, Fryer JP, et al. A prospective study of rapid corticosteroid elimination in simultaneous pancreas-kidney transplantation: comparison of two maintenance immunosuppression protocols: tacrolimus/mycophenolate mofetil versus tacrolimus/sirolimus. Transplantation 2002;73(2):169-77.

8. Sollinger HW, Odorico JS, Knechtle SJ, D'Alessandro AM, Kalayoglu M, Pirsch JD. Experience with 500 simultaneous pancreas-kidney transplants. Ann Surg 1998;228(3):284-96

9. Sutherland DE, Gruessner RW, Dunn DL, Matas AJ, Humar A, Kandaswamy R, et al. Lessons learned from more than 1,000 pancreas transplants at a single institution. Ann Surg 2001;233(4):463-501.

10. UNOS. Annual Report. OPTN Data Reports Online. Availabel at http://www.optn. org/data; 2003.

11. Krieger NR, Odorico JS, Heisey DM, D’Alessandro AM, Knechtle SJ, Pirsch JD, et al. Underutilization of pancreas donors. Transplantation 2003;75(8):1271-6.

12. Stratta RJ, Bennett L. Pancreas underutilization in the United States: analysis of United Network for Organ Sharing data. Transplant Proc 1997;29(8):3309-10.

13. Ris F, Toso C, Veith FU, Majno P, Morel P, Oberholzer J. Are criteria for islet and pancreas donors sufficiently different to minimize competition? Am J Transplant 2004;4(5):763-6.

14. ABTO. Transplantes realizados no Brasil. Revista Brasileira de Transplantes 2003; Ano 9(Terceiro Trimestre)

15. Boggi U, Del Chiaro M, Vistoli F, Signori S, Vanadia Bartolo T, Gremmo F, et al Pancreas transplantation from marginal donors. Transplant Proc 2004;36(3):566-8.

16. Bonham CA, Kapur S, Dodson SF, Dvorchik I, Corry RJ. Potential use of marginal donors for pancreas transplantation. Transplant Proc 1999;31(1-2):612-3.

17. Odorico JS. Underutilization of the potential for cadaver pancreas donation. Transplant Proc 1997; 29(8):3311-2.
18. Boggi U, Vistoli F, Del Chiaro M, Signori S, Croce C, Pietrabissa A, et al. Pancreas preservation with University of Wisconsin and Celsior solutions: a single-center, prospective, randomized pilot study. Transplantation 2004;77(8):1186-90.

19. Nghiem DD, Olson PR, Ormond D. The "fatty pancreas allograft": anatomopathologic findings and clinical experience. Transplant Proc 2004;36(4):1045-7.

20. Rhein T, Metzner R, Uhlmann D, Serr F, Caca K, Weinert D, et al. Pediatric donor organs for pancreas transplantation: an underutilized resource? Transplant Proc 2003;35(6):2145-6

21. Perosa M, Genzini T, Gullo Neto S, Caravatto PPP et al. Transplante de Pâncreas com Órgãos de Doadores Limítrofes. JBT 5(1):8-11, 2002

22. Hesse UJ, Sutherland DE. Influence of serum amylase and plasma glucose levels in pancreas cadaver donors on graft function in recipients. Diabetes 1989;38 Suppl 1:1-3.

23. Hesse UJ, Gores PF, Sutherland DE. Serum amylase and plasma glucose levels in pancreas cadaver donors: correlation with functional status of the pancreatic graft. Transplant Proc 1989;21(1 Pt 3):2765-6.

24. Hesse UJ, Najarian JS, Sutherland DE. Amylase activity and pancreas transplants. Lancet 1985;2(8457):726.

25. Masson F, Thicoipe M, Gin H, de Mascarel A, Angibeau RM, Favarel-Garrigues $\mathrm{JF}$, et al. The endocrine pancreas in brain-dead donors. A prospective study in 25 patients. Transplantation 1993;56(2):363-7.

26. Morel P, Gillingham KJ, Schlumpf R, Chau C, Moudry-Munns K, Payne WD, et al. Effect of simultaneous liver retrieval, retrieval team, and preservation time on cadaver whole-organ, bladder-drained pancreatic allograft survival rates. Transplant Proc 1991;23(1 Pt 2):1640-2.

27. Ames SA, Kisthard JK, Smith JL, Piper JB, Corry RJ. Successful combined hepatic and pancreatic allograft retrieval in donors with a replaced right hepatic artery. Surg Gynecol Obstet 1991;173(3):216-22.

28. Dunn DL, Morel P, Schlumpf R, Mayoral JL, Gillingham KJ, Moudry-Munns KC, et al. Evidence that combined procurement of pancreas and liver grafts does not affect transplant outcome. Transplantation 1991;51(1):150-7.

29. Dunn DL, Schlumpf RB, Gruessner RW, Morel P, Mayoral JL, Payne WD, et al. Maximal use of liver and pancreas from cadaveric organ donors. Transplant Proc 1990;22(2):423-4.

30. Kim SC, Lee SG, Han DJ, Lee YJ, Kwon TW, Park KM, et al. Combined procurement of liver and pancreas does not influence early graft function and survival. Transplant Proc 1996;28(3):1882-4

31. Perosa M, Genzini T, Ferreira CC, Gil AO, Engler-Pinto P, Gama-Rodrigues JJ. A welcome arterial variation for pancreas transplantation. Transplant Proc 1999;31(5):2148-9.

32. Hesse UJ, Troisi R, Maene L, de Hemptinne B, Pattyn P, Lameire N. Arterial reconstruction in hepatic and pancreatic allograft transplantation following multiorgan procurement. Transplant Proc 2000;32(1):109-10.

33. Sansalone CV, De Carlis L, Rondinara GF, Colella G, Rossetti O, Slim AO, et al. Right hepatic artery replacement from superior mesenteric artery in combined liver-whole pancreas procurement. Technical problems and liver graft artery reconstruction. Transplant Proc 1994;26(6):3537-9. 
34. Imagawa DK, Olthoff KM, Yersiz H, Shackleton CR, Colquhoun SD, Shaked A, et al. Rapid en bloc technique for pancreas-liver procurement. Improved early liver function. Transplantation 1996;61(11):1605-9.

35. Squifflet JP, de Hemptinne B, Gianello P, Balladur P, Otte JB, Alexandre GP. A new technique for en bloc liver and pancreas harvesting. Transplant Proc 1990;22(4):2070-1.

36. Dodson F, Pinna A, Jabbour N, Casavilla A, Khan F, Corry R. Advantages of the rapid en bloc technique for pancreas/liver recovery. Transplant Proc 1995;27(6):3050.

37. Pinna AD, Dodson FS, Smith CV, Furukawa H, Sugitani A, Fung JJ, et al. Rapid en bloc technique for liver and pancreas procurement. Transplant Proc 1997;29(12):647-8.

38. Boggi U, Vistoli F, Del Chiaro M, Signori S, Pietrabissa A, Costa A, et al. A simplified technique for the en bloc procurement of abdominal organs that is suitable for pancreas and small-bowel transplantation. Surgery 2004;135(6):629-41.

39. Stratta RJ. Donor age, organ import, and cold ischemia: effect on early outcomes after simultaneous kidney-pancreas transplantation. Transplant Proc 1997;29(8):3291-2.

40. Basadonna GP, Auersvald LA, Oliveira SC, Friedman AL, Lorber MI. Pancreas after kidney transplantation: HLA mismatch does not preclude success. Transplant Proc 1997;29(1-2):667.

41. Knight RJ, Bodian C, Rodriguez-Laiz G, Guy SR, Fishbein TM. Risk factors for intraabdominal infection after pancreas transplantation. Am J Surg 2000;179(2):99-102.

42. Teraoka S, Nakagawa Y, Nakamura M, Hirotani S, Tohjinbara T, Tomonaga O, et al. Assessment of viability of pancreas transplants from non-heart-beating cadaver. Transplant Proc 1998;30(2):301-3.

43. Tojimbara T, Teraoka S, Babazono T, Sato S, Nakamura M, Kimikawa M, et al Strategy in combined pancreas and kidney transplantation from non-heart-beating cadaver donors. Transplant Proc 1999;31(5):2008-9.
44. Tojimbara T, Teraoka S, Babazono T, Sato S, Nakamura M, Hoshino T, et al. Longterm outcome after combined pancreas and kidney transplantation from non-heartbeating cadaver donors. Transplant Proc 1998;30(7):3793-4.

45. Markmann JF, Deng S, Desai NM, Huang X, Velidedeoglu E, Frank A, et al. The use of non-heart-beating donors for isolated pancreatic islet transplantation. Transplantation 2003;75(9):1423-9.

46. Hesse UJ, Berrevoet F, Pattyn P, Vanholder R, de Hemptinne B. Donor parameters of pancreas grafts processed for islet or beta-cell isolation and transplantation. Transplant Proc 1997;29(4):2259.

47. Gores PF, Viste A, Hesse UJ, Moudry-Munns KC, Dunn DL, Sutherland DE. The influence of donor hyperglycemia and other factors on long-term pancreatic allograft survival. Transplant Proc 1990;22(2):437-8.

48. Gores PF, Gillingham KJ, Dunn DL, Moudry-Munns KC, Najarian JS, Sutherland DE. Donor hyperglycemia as a minor risk factor and immunologic variables as major risk factors for pancreas allograft loss in a multivariate analysis of a single institution's experience. Ann Surg 1992;215(3):217-30.

49. Barone GW, Henry ML, Elkhammas EA, Tesi RJ, Ferguson RM. Whole-organ transplant of an annular pancreas. Transplantation 1992;53(2):492-3.

50. Romagnoli J, Papalois VE, Hakim NS. Transplantation of an annular pancreas with enteric drainage. Int Surg 1998;83(1):36-7.

51. White JC, Shaver TR, Kocandrle V. Simultaneous kidney-pancreas transplantation using a horseshoe kidney. Transpl Int 1993;6(5):302-3.

52. Eliaschewitz FG; Aita CA; Genzini T et al. First Brazilian pancreatic islet transplantation in a patient with type 1 diabetes mellitus.Transplant Proc 36(4): 1117-8, 2004. 